\title{
MiR-99a Enhances the Radiation Sensitivity of Non-Small Cell Lung Cancer by Targeting mTOR
}

\author{
Hang Yin ${ }^{a}$ Jianqun Ma ${ }^{b} \quad$ Lin Chen $^{a} \quad$ Shiqi Piao ${ }^{a} \quad$ Yu Zhang ${ }^{a} \quad$ Siliang Zhang \\ Hongyu Maa Yang Lia Yuanyuan $\mathrm{Qu}^{a} \quad$ Xiaoyuan Wang ${ }^{\mathrm{b}}$ Qingyong Xua \\ The Department of Radiotherapy Oncology, Harbin Medical University Cancer Hospital, Harbin, \\ Heilongjiang Province, ${ }^{\mathrm{b} T h e}$ Department of Oncology, Harbin Medical University Cancer Hospital, \\ Harbin, Heilongjiang Province, China
}

\section{Key Words}

Microrna-99a • Non-small cell lung cancer (NSCLC) • Mammalian target of rapamycin (mTOR) - Radiosensitivity

\begin{abstract}
Background/Aims: Radiation therapy is an important and effective modality for the treatment of non-small cell lung cancer (NSCLC). MicroRNAs (miRNAs) are crucial post-transcriptional regulators that are involved in numerous important biologic processes. However, their potential involvement in radiation sensitivity remains unknown. Materials: We performed integrated analysis of miRNA expression in NSCLC using The Cancer Genome Atlas datasets. miR-99a was found to be significantly upregulated in cancer tissue and regulated cell survival. Cell culture was used to assess the role of miR-99a in radiation sensitivity. We then used flow cytometry to examine the effects of miR-99a on the cell cycle and apoptosis in cells exposed to radiation. To identify gene targets of miR-99a, a bioinformatics approach was adopted, and the findings of this analysis were verified using luciferase reporter assays. Finally, an in vivo study was conducted to examine the effect of miR-99a on tumor volume in an NSCLC mouse model undergoing radiation therapy. Results: miR-99a was significantly upregulated in radiationsensitive A549 cells compared with radiation-resistant A549 cells. miR-99a overexpression was shown to enhance radiosensitivity, while inhibition of miR-99a resulted in radioresistance of NSCLC cell lines in vitro and in vivo. In addition, by bioinformatics software analysis and luciferase assays, mammalian target of rapamycin (mTOR) was identified as a direct target of miR-99a. Furthermore, AZD2014, an inhibitor of mTOR, enhanced radiosensitivity and apoptosis in NSCLC cell lines, while mTOR overexpression resulted in radioresistance and cell survival from miR-99a-induced cell apoptosis. Moreover, miR-99a overexpression further increased the efficacy of radiation therapy in an NSCLC xenograft mouse model, and miR-99a
\end{abstract}

H. Yin, J. Ma, Q. Xu and X. Wang contributed equally to this work.

Qingyong Xu

and Xiaoyuan Wang,
Department of Radiotherapy Oncology, Harbin Medical University Cancer Hospital,

Harbin 150040, Heilongjiang Province (China)

Tel. 86-451-86298517. E-Mail xuqingyonghmu@163.com, 346650985@qq.com 


\section{Cellular Physiology Cell Physiol Biochem 2018;46:471-481

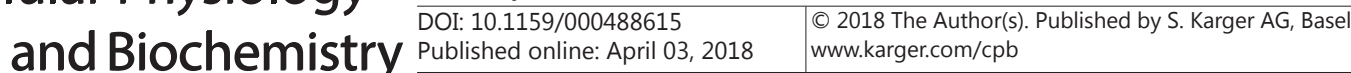 \\ Yin et al.: miR-99a Enhances the Radiation Sensitivity}

and mTOR expression was significantly inversely correlated. Conclusions: Altogether, these data suggested miR-99a functions as a tumor suppressor that has a critical role in regulating radiosensitivity of NSCLC by targeting the MTOR signaling pathway.

(C) 2018 The Author(s)

Published by S. Karger AG, Basel

\section{Introduction}

Lung cancer is the most common cause of cancer-related death worldwide [1]. Non-small cell lung cancer (NSCLC) accounts for approximately 85\% of lung cancer cases. Therefore, the identification of methods to improve the survival rate of patients with NSCLC has become an important task [2]. At present, treatment for NSCLC includes surgery, chemotherapy, and radiotherapy [3], of which radiotherapy is the main approach [4]. Nevertheless, despite the great efforts that have been exerted to develop radiation therapies for NSCLC patients, the 5 -year overall survival rate still remains less than $15 \%[1,5]$. Local recurrence is a common clinical problem and is the primary contributor to the poor prognosis of a large number of patients [6]. One of the major reasons for local recurrence is the development of radiation resistance [7]. Therefore, clarification of the mechanism underlying radiation resistance and the establishment of a valid prognostic factor and therapeutic target are clinically important.

MicroRNAs (miRNAs) are a class of small, single-stranded, endogenous non-coding RNA consisting of 19-25 nucleotides [8]. miRNAs interact with the 3 '-untranslated region (UTR) of the mRNA of target genes to promote mRNA degradation and/or inhibit protein translation [9]. With the expansion of efforts to study miRNA expression, abnormal expression patterns of miRNAs have been identified as unique clinical biomarkers that are relevant for diagnosis, stage, prognosis, and response to treatment [10]. Additionally, miRNAs can also regulate radiation sensitivity by activating oncogenic pathways or suppressing tumor suppressor gene pathways or protein expression [11]. Differences in miRNA expression are associated with the efficacy of radiotherapy and may act as biomarkers for predicting the efficacy of radiotherapy [12]. Furthermore, miRNA (miR)-99a has been identified as an important prognostic factor that regulates radiation sensitivity in a variety of different cancers, such as renal cancer and prostate cancer [13-15]. With regard to NSCLC, miR-99a expression is upregulated in lung cancer tissues as compared with matched normal tissues [16,17]. These results suggest that miR-99a might play a critical role in NSCLC radiation sensitivity, but the underlying mechanism remains unknown.

Mammalian target of rapamycin (mTOR), as a downstream gene of the PI3K/AKT pathway, has been a hot target for cancer research in recent years [18]. This pathway has been implicated in the regulation of angiogenesis, cell survival, proliferation, and metastasis [19]. Furthermore, mTOR also plays an important role in radiation sensitivity, and an inhibitor of mTOR has been shown to enhance radiosensitivity [20-22]. However, the detail mechanisms remain poorly defined.

In this study, we found that miR-99a was significantly upregulated in radiationsensitive A549 cells compared with radiation-resistant A549 cells. Specifically, miR-99a overexpression enhanced radiosensitivity and apoptosis by directly targeting mTOR in vitro and in vivo. According to our results, we propose that miR-99a is a potential miRNA for modulating the radiation sensitivity of NSCLC.

\section{Materials and Methods}

To analyze the expression of miR-99a and mTOR in NSCLC, we used the datasets in TCGA (https:// cancergenome.nih.gov). We queried TCGA for miR-99a and mTOR gene and filtered the results by selecting NSCLC and the Lung Cancer vs. Normal Analysis database. The results were displayed using a box chart, and Student's t-test was used to calculate $P$-values. 


\section{Cellular Physiology Cell Physiol Biochem 2018:46:471-481 \begin{tabular}{l|l} 
DOI: 10.1159/000488615 & $\begin{array}{l}\text { O } 2018 \text { The Author(s). Published by S. Karger AG, Basel } \\
\text { www.karger.com/cpb }\end{array}$
\end{tabular} \\ Yin et al.: miR-99a Enhances the Radiation Sensitivity}

Cell culture

The normal lung epithelial cell line HBE and NSCLC cell lines A549, H1650, and H1299 were obtained from Heilongjiang Cancer Institute (Harbin, China). The cells were cultured in RPMI-1640 supplemented with $10 \%$ fetal bovine serum at $37^{\circ} \mathrm{C}$ with $5 \% \mathrm{CO}_{2}$.

To generate radiation-resistant cells, A549 cells were firstly grown to approximately $90 \%$ confluence and then exposed to irradiation at $2.0 \mathrm{~Gy} /$ fraction using $6 \mathrm{MV}$ X-rays generated by an accelerator provided by Harbin Medical University Cancer Hospital, and the final dose was 64 Gy. The selected radioresistant cell line was named A549-R.

\section{Real-time quantitative PCR ( $q P C R$ )}

RNA from cells and tissues was isolated using the TRIzol reagent (Invitrogen). The isolated RNA was then reverse transcribed into cDNA using a cDNA Synthesis Kit (Fermentas, Foster City, CA, USA). Levels of miR-99a and U6 expression were determined using a SYBR Premix Ex-Taq II Kit and an ABI 7500 machine according to the manufacturer's instructions. The $2^{-\Delta \Delta \mathrm{Ct}}$ method was used to calculate expression levels.

\section{Western blot analysis}

The cells were lysed in a lysis buffer, and $50 \mu \mathrm{g}$ protein from each sample was resolved by sodium dodecyl sulfate-polyacrylamide gel electrophoresis (10\% gels) and transferred to a polyvinylidene fluoride membrane. The membrane was probed with anti-mTOR, anti-phosphorylated (p)-mTOR, anti-Bcl-2, anticyclin D1, anti-p-Smad3, anti-p-S6, and anti-S6 antibodies (Cell Signaling). Antibody detection was performed using an ECL Western Blotting Detection System. Band intensities were normalized to the glyceraldehyde 3-phosphate dehydrogenase loading control.

\section{Plasmid transfection}

The miR-99a mimics, miR-99a inhibitor, mTOR plasmid, and controls were purchased from Santa Cruz Biotechnology. Lipofectamine 2000 (Invitrogen) was used according to the manufacturer's instructions. The transfected cells were collected for analyses at $24 \mathrm{~h}$ after transfection.

\section{Dual luciferase reporter assay}

We seeded $1.0 \times 10^{5} \mathrm{H} 1299$ cells/well in a 24-well plate. Transcriptional activity was detected by cotransfection of mTOR-3'-UTR-wild-type or mTOR-3'-UTR-mutant and miR-99a or miR-negative control (NC) using Lipofectamine 2000 (Invitrogen) as per the manufacturer's instructions. Luciferase activity was assayed at $24 \mathrm{~h}$ post-transfection using the Dual Luciferase Reporter Assay System (Promega) and normalized to Renilla luciferase activity.

\section{Colony formation assay}

The cells were plated at a low density in 6-well plates overnight. The cells were then irradiated with 0 , $2,4,6$, 8, or 10 Gy X-rays and then incubated for 14 days. After fixation with methanol, the cells were stained with crystal violet and counted. All experiments were conducted at least three times. Survival rates were evaluated relative to 0 Gy radiation-treated controls. A single-hit multi-target model was used to analyze the data. A survival curve was plotted for each group as the log of the survival fraction versus radiation dose using GraphPad Prism 5.0 software.

\section{Flow cytometry}

The cells were irradiated with 6 Gy X-rays after transfection with miR-99a mimics, inhibitor, or NC. For the analysis of apoptosis, the collected cells were pooled and stained with $5 \mu \mathrm{L}$ annexin V-FITC and $10 \mu \mathrm{L}$ propidium iodide (PI) using an Annexin V FITC Apoptosis Detection Kit I (556547; BD Biosciences) according to the manufacturer's instructions and analyzed on a BD Accuri C6. For cell cycle analysis, the cells were washed with cold phosphate-buffered saline (PBS) and fixed with $70 \%$ ethanol overnight at $-20^{\circ} \mathrm{C}$. The fixed cells were washed with PBS for $10 \mathrm{~min}$ and processed for PI/RNase staining. The cell cycle stage of each sample was evaluated by flow cytometry (Becton-Dickinson). 
Immunohistochemistry

Tissue sections were immersed in minocycline-ethylenediaminetetraacetic acid, heated in a steamer at $100^{\circ} \mathrm{C}$ for $15 \mathrm{~min}$, and incubated in methanol for $15 \mathrm{~min}$. The slides were incubated with an anti-mTOR primary antibody, stained using 3, 3'-diaminobenzidine, and counterstained with hematoxylin. Relative staining intensity was judged according to the percentage of immunoreactive cells.

\section{In vivo radiation sensitivity}

The animal experiments were approved by the Scientific Investigation Board of the Tumor Hospital of Harbin Medical University. We purchased 4-6-week-old nude mice (male) from the Shanghai Experimental Animal Center, and they were bred in the Animal Center of the affiliated Tumor Hospital of Harbin Medical University. The mice were injected with $4.0 \times 10^{6} \mathrm{~A} 549$ cells. After 8 days, the transplanted nude mice were randomly divided into two groups. Agomir-miR-99a or agomir-NC (RiboBio Co., Ltd., Guangzhou, China) was injected directly into the implanted tumor at a dose of $1 \mathrm{nmol}$ per mouse every 4 days for a total of 7 times. Tumor volume $(\mathrm{V})$ was calculated with the equation: $\mathrm{V}=\left(\mathrm{W}^{2} \times \mathrm{L}\right) \times 0.5$. When the volume of tumors reached 500 to $1000 \mathrm{~mm}^{3}$ (at approximately 20 days after injection), the mice were exposed to $10 \mathrm{~Gy} \mathrm{X}$-rays once every 3 days for a total of 2 exposures: applicator size, $15 \times 15$ $\mathrm{cm}$; final radiation field for the tumor was $1 \mathrm{~cm}$ greater than the edge of the tumor with lead block shielding, covering a $1 \mathrm{~cm}$ bolus on the surface of the tumor to enhance the subcutaneous radiation dose; source to skin distance, $100 \mathrm{~cm}$; and electron beam irradiation, $9 \mathrm{MeV}$. Seven weeks later, the mice were sacrificed and the tumors and lungs were harvested and fixed in formalin.

\section{Statistical analysis}

SPSS 18.0 and GraphPad Prism software were used for statistical analyses. Statistical significance between two groups was calculated by Student's t-test. Statistical significance is indicated using asterisks: ${ }^{*} P<0.05$ and ${ }^{* *} P<0.01$.

\section{Results}

miR-99a expression is significantly downregulated in human lung cancer tissues

To determine the role of miR-99a in NSCLC development, we analyzed miRNA and gene expression profiles using 999 NSCLC samples and 91 adjacent normal lung tissues obtained from the TCGA database. miR99a was found to be significantly downregulated in NSCLC tissues compared with adjacent normal

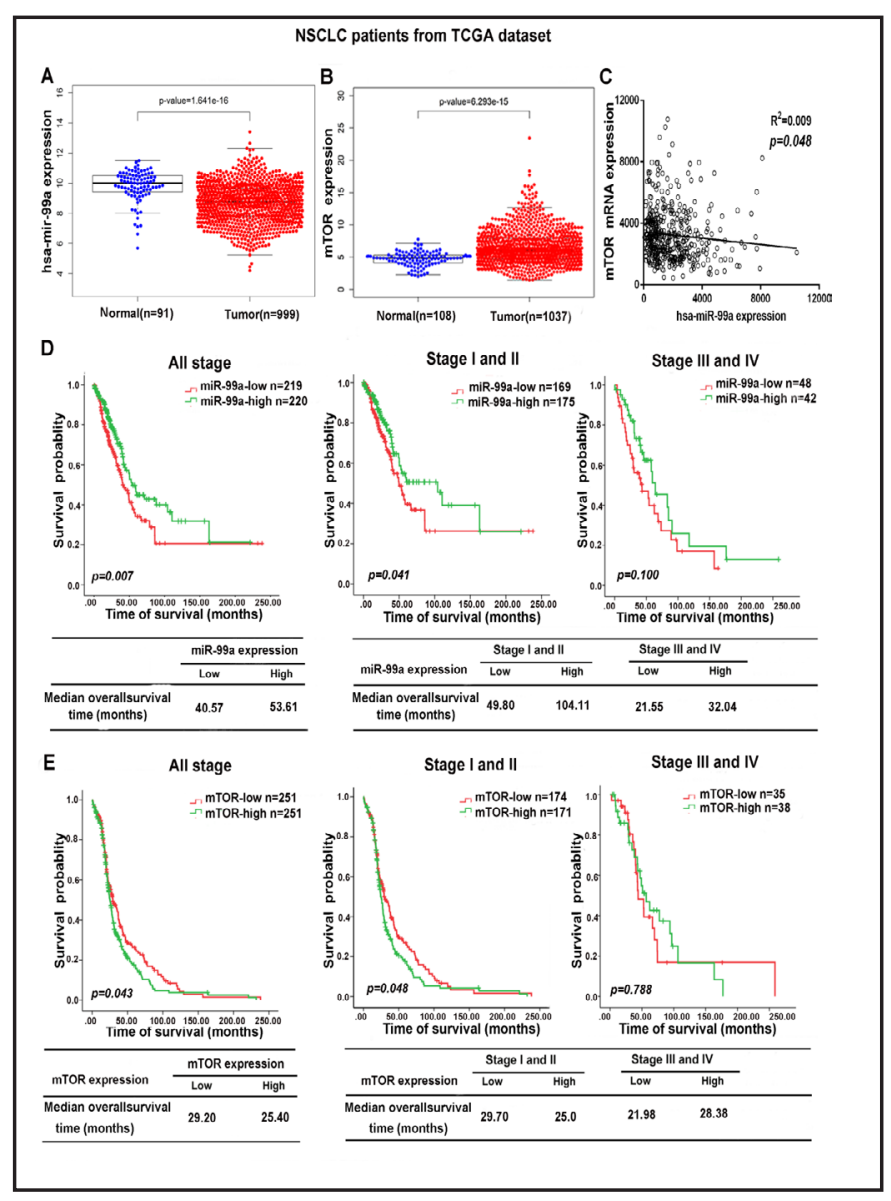

Fig. 1. The expression of miR-99a and mTOR in NSCLC patients of TCGA database. (A) The expression of miR-99a was significantly downregulated in human lung cancer tissues compared with adjacent normal tissues.(B) The expression of mTOR was significantly upregulated in human lung cancer tissues compared with adjacent normal tissues. (C) The expression of miR-99a was inversely related with mTOR in NSCLC patients. (D) The high expression of miR-99a had a better median overall survival. (E) The high expression of MTOR had a worse median overall survival. 
Fig. 2. MiR-99a sensitizes NSCLC cells to radiation therapy. (A) q-PCR analysis to quantify the expression levels of miR-99a in HBE, A549-R, A549 and H1650 cell lines. U6 was used for normalization. (B) A549 and A549-R cells were transfected with anti-NC, miR-99a inhibitors, miR-NC, miR-99a mimics and the expression changes of miR99a were analyzed by real-time PCR. (C, D) Survival fraction of A549-anti99a was higher than A549-anti-NC and A549-untreated, A549R-miR-99a was lower than A549R-miR-NC and A549Runtreated cells. (E) Flow cytometric analysis of cell cycle in A549 and A549R were determined after transfected miR99a mimics, miR-controls, miR-99a inhibitors or negative controls combined with 6Gy irradiation.(F) Flow cytometric analysis of apoptosis in A549-R and A549 were determined after transfected miR-99a mimics, miR-NC, miR-99a inhibitors or negative controls combined with 6Gy irradiation.

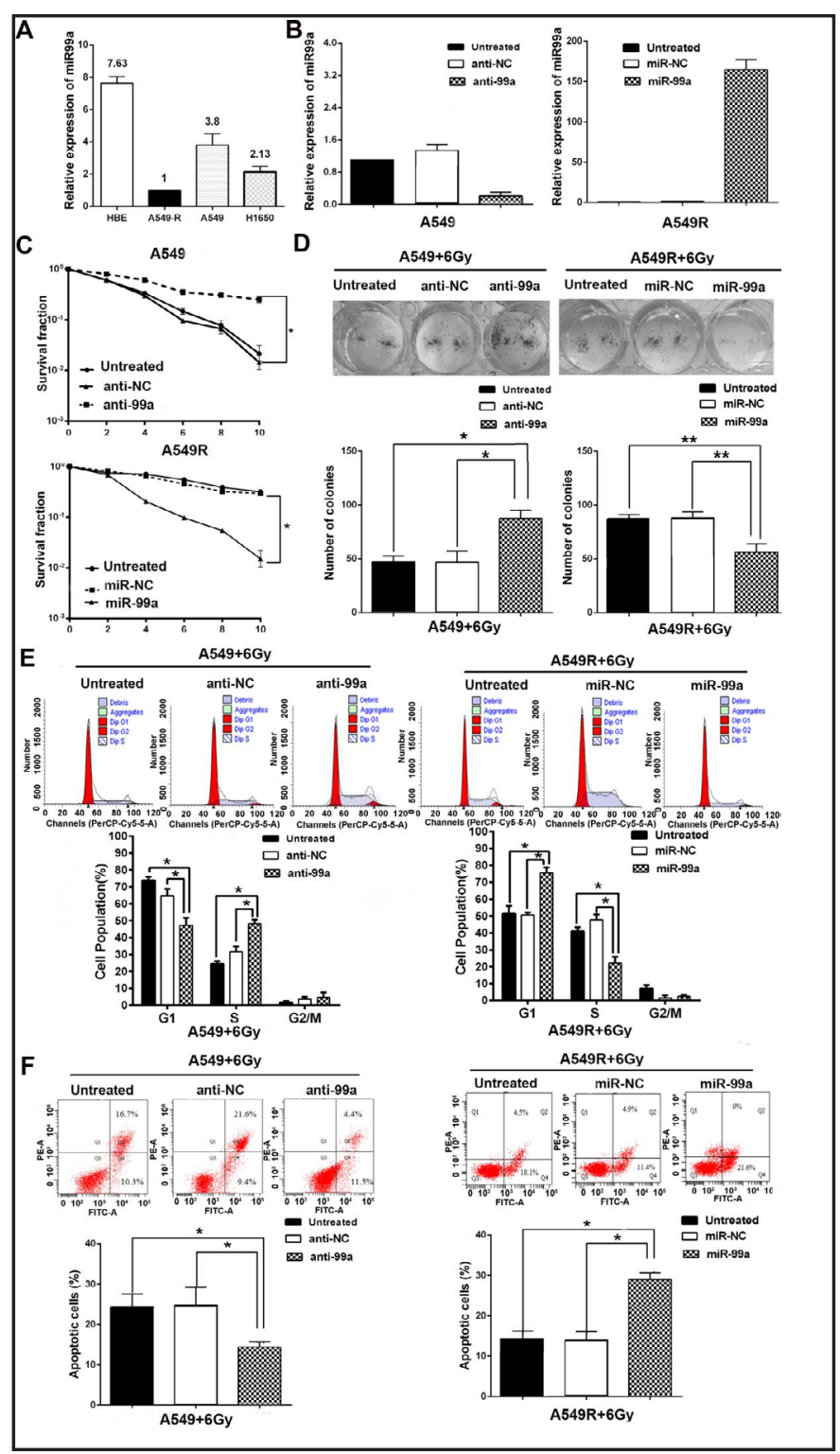

lung tissues $(P<0.05$, Fig. 1A). Furthermore, NSCLC patients with high miR-99a expression had better overall survival than those with low miR-99a expression $(P<0.05$, Fig. 1D).

miR-99a sensitizes NSCLC cells to radiation therapy

To explore the role of miR-99a, we analyzed the expression levels of miR-99a in HBE normal lung epithelial cells, H1650 cells, and A549 cells. qPCR analysis showed that miR-99a expression was significantly lower in H1650 and A549 cells than in HBE cells (Fig. 2A).

Then, we generated a radiation-resistant NSCLC cell line to investigate the association between miR-99a expression and radiation sensitivity. miR-99a expression levels were significantly downregulated in the A549-R cells compared with the A549 cells (Fig. 2A). Thus, these results suggested that miR-99a may contribute to radiation resistance in NSCLC cells.

Following this analysis, to assess further the role of miR-99a in the radiation sensitivity of NSCLC cells, A549 cells were transfected with miR-NC and miR-99a inhibitors. By contrast, A549-R cells were transfected with miR-NC and miR-99a-mimics, and the expression levels of miR-99a were verified by qPCR (Fig. 2B). In addition, A549, A549-R, and transfected cells 


\section{Cellular Physiology Cell Physiol Biochem 2018;46:471-481 \begin{tabular}{l|l} 
DOI: 10.1159/000488615 & a 2018 The Author(s). Published by S. Karger AG, Basel \\
www.karger.com/cpb
\end{tabular} \\ Yin et al.: miR-99a Enhances the Radiation Sensitivity}

Fig. 3. MTOR is a direct target of miR99a-5p. (A) Venn diagrams showed the number of genes identified as potential targets of miR-99a using four bioinformatics methods of miRanda, PITA, Targetscan and miRWalk. (B) MiR-99a significantly decreased the luciferase activity in the wild type mTOR 3 '-UTR cells $(\mathrm{p}<0.05)$ and no siginificant effects in the mutant type mTOR 3'-UTR cells. (C) Western blot showed the levels of mTOR were sigin-

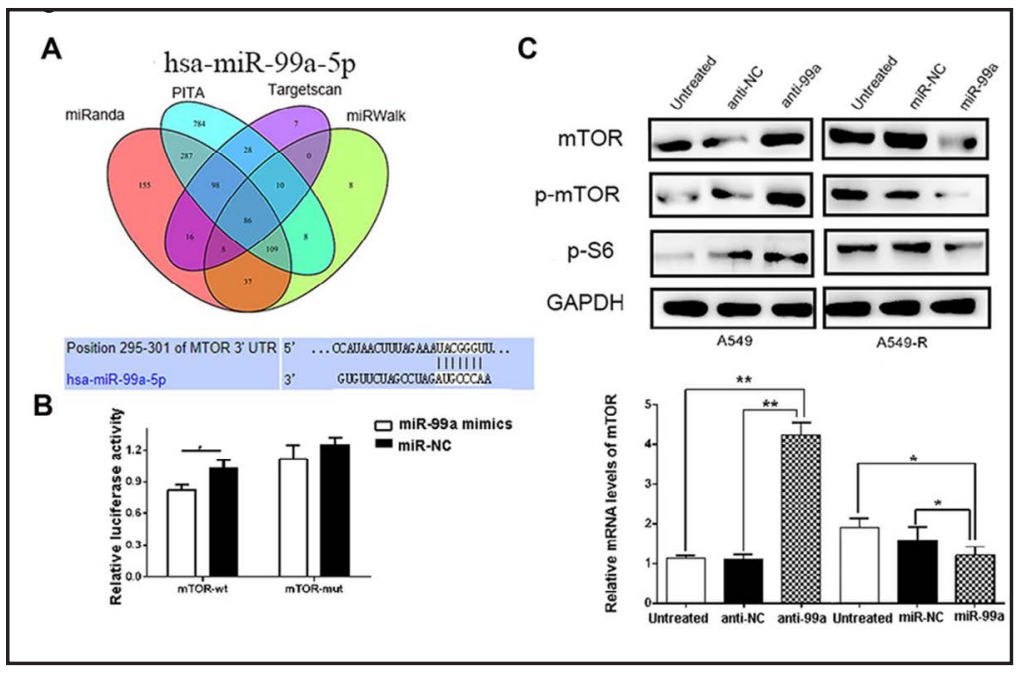
ificantly decreased in A549 cells compared with A549-R cells. After transfected miR-99a mimics, the expression of mTOR, p-mTOR and p-S6 decreased in A549-R cells. By contrast, after transfected miR-99a inhibitors, the expression of mTOR, p-mTOR and p-S6 increased in A549 cells.

were exposed to various doses of radiation $(0,2,4,6$, 8, or $10 \mathrm{~Gy})$, and we found that miR99a enhanced the effect of radiation in A549-R cells and reduced its effect in A549 cells (Fig. $2 \mathrm{C}$ and $\mathrm{D}$ ). These results further suggested that the upregulated expression of miR-99a may enhance radiation sensitivity.

Radiation sensitivity is often related to cell cycle arrest and apoptosis; therefore, we used flow cytometry analysis to study the effect of miR-99a on apoptosis and the cell cycle in irradiated NSCLC cells. Cells transfected with the miR-99a mimics showed a higher percentage of cells in the G1 stage than the miR-NC and untreated groups of A549-R cells $(P<0.05)$ and a lower percentage of cells in the $\mathrm{S}$ stage $(P<0.05$, Fig. 2E). By contrast, the percentage of anti-miR-99a-A549 cells in the G1 phase was decreased and the percentage of these cells in the $\mathrm{S}$ phase was increased compared with the anti-NC and untreated groups $(P<0.05$, Fig. 2E). Analysis of apoptosis showed that miR-99a combined with irradiation increased the ratio of apoptotic A549-R cells $(P<0.05$, Fig. 2F). However, blocking miR99 a combined with irradiation significantly decreased the ratio of apoptotic A549 cells $(P$ $<0.05$, Fig. 2F). Therefore, these data suggested that miR-99a could increase the radiation sensitivity of NSCLC cells, which may have been caused by the induction of cell arrest in the G1 phase and increased apoptosis when miR-99a was combined with radiation treatment.

\section{mTOR is a direct target of miR-99a}

To explore the mechanisms underlying the effects of miR-99a on cell proliferation and radiation sensitivity in NSCLC, potential target genes were analyzed by using four bioinformatics approaches: miRanda, PITA, TargetScan, and miRWalk. All four approaches localized mTOR at the center of a net comprising 86 genes (Fig. 3A) and predicted that miR99a may target the $3^{\prime}$-UTR region of MTOR.

Next, we searched the TCGA database again and found that mTOR expression was upregulated in NSCLC tissues compared with normal lung tissues $(P<0.01$, Fig. 1B). Additionally, for TCGA NSCLC patients, miR-99a expression was inversely correlated with mTOR levels, and mTOR expression was correlated with survival $(P<0.05$, Fig. $1 \mathrm{C}$ and E).

To confirm our speculation further, we generated luciferase reporters for miR-99a and mTOR. H1299 cells were transfected with wild-type or mutant mTOR. miR-99a was found to significantly decrease luciferase activity in cells transfected with wild-type mTOR 3'-UTR $(P<0.05)$, while no significant effect was observed in cells transfected with mutant mTOR 3'-UTR (Fig. 3B). 
In addition, we detected the protein and mRNA levels of $\mathrm{mTOR}$ and components of its downstream pathway, including p-mTOR and p-S6. The levels of mTOR were found to be significantly decreased in A549 cells compared with A549-R cells. After transfection with miR-99a mimics, the levels of mTOR, p-mTOR, and p-S6 were decreased in A549-R cells. By contrast, after transfection with miR-99a inhibitors, the levels of mTOR, p-mTOR, and p-S6 were increased in A549 cells (Fig. 3C). Therefore, these results showed that MTOR was a target of miR-99a, as predicted.

mTOR plays
a crucial role
in the miR-
$99 a-m e d i a t e d$
rad i a $t$ i o $n$
sensitivity of
NSCLC cell lines
W

i n ve s t i g a t e d whether mTOR was responsible for the radiation sensitivity of NSCLC cells regulated by $\mathrm{miR}-$ 99a. When mTOR was inhibited in anti-miR-99a-A549 cells by AZD2014, the cells became more sensitive to radiation than control cells (Fig. 4A) and the levels of anti-apoptosis markers were reduced (Fig. 4D). When mTOR was transfected into miR-99aA549-R cells, the cells became more resistant to radiation therapy than control cells and

Fig. 4. MTOR played a crucial role in miR-99a mediated radiation sensitivity of NSCLC cell lines. (A) Clonogenic survival analysis of 0, 2, 4, 6, 8, 10 Gy in A549anti-NC, A549-anti-99a, A549-anti-99a+AZD, A549R-miR-NC, A549R-miR-99a, A549R-miR-99a+mTOR cell lines. (B) Flow cytometric analysis of apoptosis in A549-anti-NC, A549-anti-99a, A549-anti-99a+AZD, A549R-miR-NC, A549RmiR-99a, A549R-miR-99a+mTOR cell lines combined with 6Gy irradiation. (C) Flow cytometric analysis of cell cycle in A549-anti-NC, A549-anti-99a, A549anti-99a+AZD, A549R-miR-NC, A549R-miR-99a, A549R-miR-99a+mTOR cell lines combined with 6Gy irradiation. (D) Western blot detection of mTOR, Bcl2, CyclinD1, p-S6, S6 expression in A549-anti-NC, A549-anti-99a, A549-anti99a+AZD, A549R-miR-NC, A549R-miR-99a, A549R-miR-99a+mTOR cell lines. GAPDH was used as an internal control.

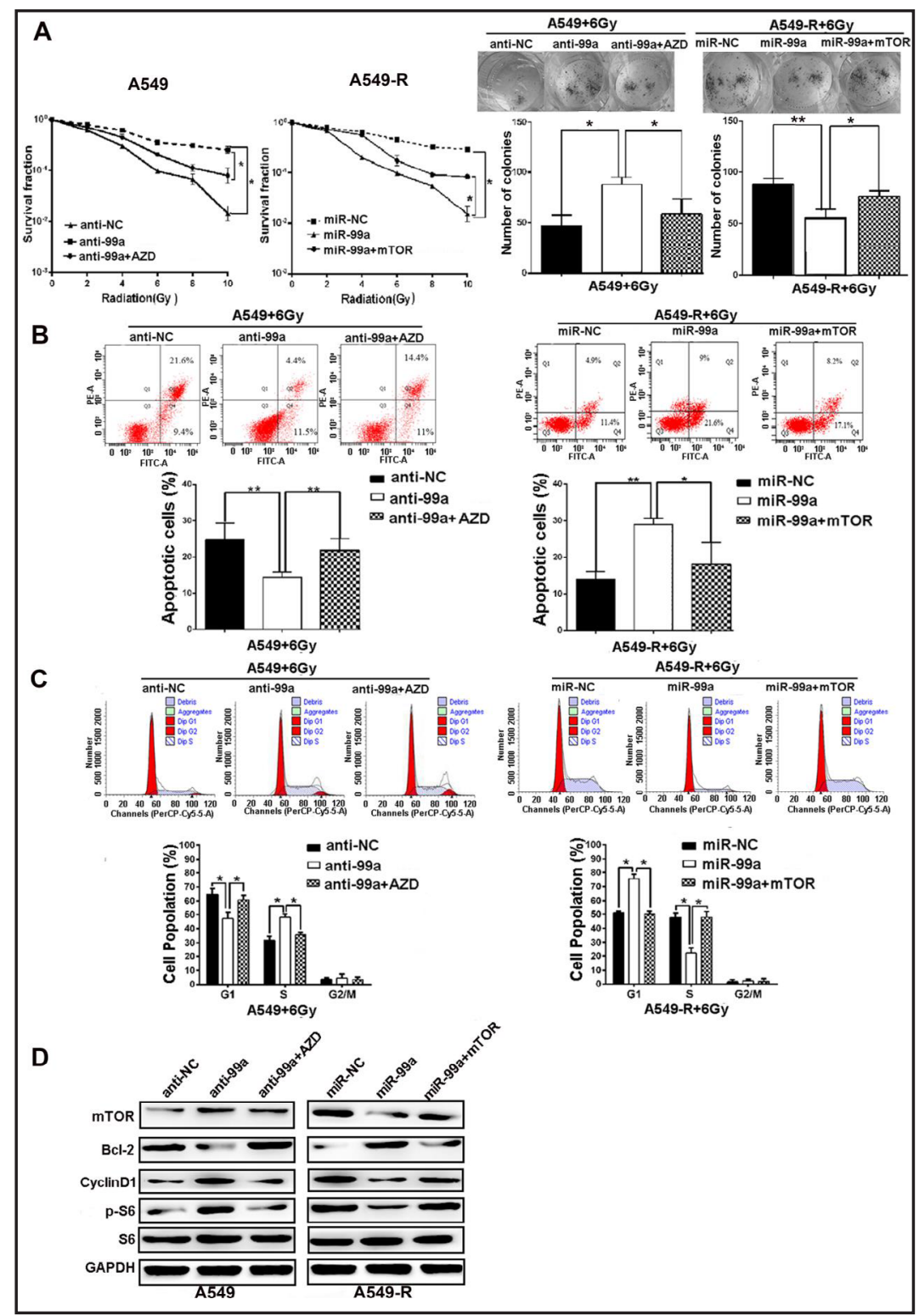


the expression of antiapoptosis markers was increased (Fig. 4A-D).

\section{miR-99a regulates radiosensitivity in vivo}

To analyze whether miR-99a could influence NSCLC radiosensitivity in vivo, we used an NSCLC mouse model. miR-99a combined with irradiation was observed to significantly decrease tumor volume compared with the miRNC group (Fig. 5A, B, and $C$ ). Furthermore, immunohistochemical analysis indicated that mTOR expression was decreased in the miR99a agomir group compared with the NC
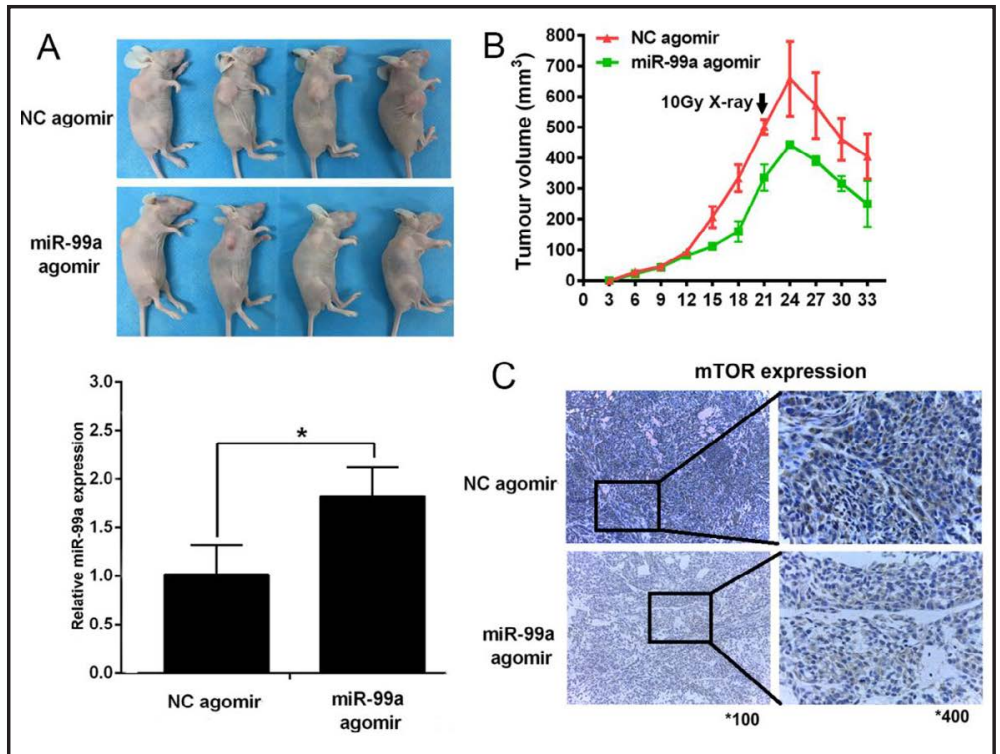

Fig. 5. MiR-99a regulates radiosensitivity in vivo. (A) Mice were injected with NC-agomir and miR99a-agomir which were named as NC agomir group and miR-99a agomir group respectively. (B) MiR-99a significantly decreased the tumor volumes of mouse combined irradiation than miR-NC group. (C) MTOR expression of miR-99a agomir group was decreased than $\mathrm{NC}$ group in the tissue. group (Fig. 5D). These in vivo findings further proved that miR-99a enhanced the radiation sensitivity of NSCLC by targeting mTOR.

\section{Discussion}

Radiation therapy is known to be an important approach for the treatment of NSCLC, but the obstacle of radiation resistance has a negative effect on the survival of patients with NSCLC [23]. Recently, several studies have suggested that miRNAs play a role in the radiation resistance of NSCLC by interacting with tumor-related genes [24, 25]. Hence, it is important to characterize tumor-related miRNAs and their tumor-relevant gene targets [26]. Furthermore, by exploring the biological significance of these genes, we can identify novel therapeutic targets for enhancing the efficacy of radiation therapy and improve the survival of these patients.

miR-99a has been reported to be abnormally expressed in various malignant tumors and may function as a tumor suppressor. For example, downregulation of miR-99a in oral squamous cell carcinoma contributes to the growth and survival of oral cancer cells [27]. In osteosarcoma and hepatocellular cancer, miR-99a also functions as a tumor suppressor [28, 29]. In the present study, miR-99a expression was significantly downregulated in human lung cancer tissues compared with adjacent normal lung tissue and was correlated with survival, which was similar to the observations of the previous studies.

Furthermore, miR-99a has been shown to be correlated with not only tumor proliferation but also radiation sensitivity. For example, miR-99a enhances the radiosensitivity of prostate cancer cells and rectal carcinoma [14, 15]. However, whether the differential expression of miR-99a in malignant and benign NSCLC lesions can regulate radiosensitivity is still in question. Therefore, we examined differences in the expression of miRNAs in radiationsensitive and radiation-resistant NSCLC cells and found that miR-99a was overexpressed in radiation-sensitive cells. Our study also showed that miR-99a influenced the radiosensitivity 


\section{Cellular Physiology Cell Physiol Biochem 2018;46:471-481 \begin{tabular}{l|l} 
and Biochemistry Published online: April 03, 2018 & $\begin{array}{l}\text { (c) } 2018 \text { The Author(s). Published by S. Karger AG, Basel } \\
\text { www.karger.com/cpb }\end{array}$ \\
\hline POI.1159/00488615
\end{tabular} \\ Yin et al.: miR-99a Enhances the Radiation Sensitivity}

of NSCLC, and specifically, miR-99a overexpression increased radiosensitivity and the rate of apoptosis. By contrast, downregulating miR-99a expression resulted in radiation resistance.

mTOR is located downstream of the PI3K/AKT pathway and could regulate radiosensitivity by having an impact on the cell cycle and apoptosis. AZD2014 is an inhibitor of mTOR and has been shown to enhance radiation sensitivity, apoptosis, and DNA damage, and to suppress proliferation and clonogenicity [30,31]. For example, this inhibitor of the mTOR pathway enhanced the radiosensitivity of prostate cancer and glioma stem cells [32, 33]. Consistent with the role of mTOR observed in previous studies, we found that AZD2014 enhanced cellular sensitivity to radiation; by contrast, upregulating mTOR expression decreased radiation sensitivity. Furthermore, by bioinformatics software and luciferase experiments, we demonstrated that mTOR is a direct target of miR-99a, and inhibition of mTOR generated similar effects as that of ectopic miR-99a expression, whereas mTOR overexpression rescued the function of miR-99a-mediated radiosensitivity in NSCLC cells.

Radiosensitivity is affected by a complex signaling cascade and a variety of responses that include cell cycle arrest, induction of stress-response genes, DNA repair, and apoptosis [34]. In the present study, some important functional alterations in genes were observed, including Bcl-2 and cyclin D1, which are related to anti-apoptosis and the cell cycle, during miR-99a-mediated mTOR inactivation. Especially, mTOR inhibition by AZD2014 displays a consentaneous phenomenon with the effect of miR-99a. These data support the major involvement of miR-99a-mediated mTOR inactivation and increased radiosensitivity in the induction of apoptosis.

In summary, our study demonstrated that miR-99a augmented the radiosensitivity of NSCLC cells in vitro and in vivo. Furthermore, miR-99a may increase the radiosensitivity of NSCLC cells by directly targeting the mTOR signaling pathway. miR-99a and mTOR expression was negatively correlated and associated with radiosensitivity. Thus, miR-99a plays multiple tumor-suppressive roles in NSCLC cells. Our findings suggest that miR-99a and mTOR might be promising prognostic and therapeutic targets to increase radiosensitivity in NSCLC.

\section{Acknowledgements}

This work was supported by the Fundamental Research Funds for the Provincial Universities (No.2017LCZX94 to H.Y.), Haiyan Fundation of Harbin Medical Univercity Cancer Hospital (No.JJQN2017-10 to H.Y.), Jieping Wu Fundation (No.320.6799.15046 to J.M.) and Academy of Medical Sciences in Heilongjiang (No.201606 to J.M.)

\section{Disclosure Statement}

No conflict of interests exists.

\section{References}

1 Siegel RL, Miller KD, Jemal A: Cancer statistics, 2017. CA Cancer J Clin 2017;67:7-30.

2 Ou SH, Zell JA, Ziogas A, Anton-Culver H: Prognostic factors for survival of stage i nonsmall cell lung cancer patients : A population-based analysis of 19, 702 stage i patients in the california cancer registry from 1989 to 2003. Cancer 2007;110:1532-1541.

-3 Osarogiagbon RU, Lin CC, Smeltzer MP, Jemal A: Prevalence, prognostic implications, and survival modulators of incompletely resected non-small cell lung cancer in the u.S. National cancer data base. J Thorac Oncol 2016;11:e5-16.

4 Lischalk JW, Woo SM, Kataria S, Aghdam N, Paydar I, Repka MC, Anderson ED, Collins BT: Long-term outcomes of stereotactic body radiation therapy (sbrt) with fiducial tracking for inoperable stage i nonsmall cell lung cancer (nsclc). J Radiat Oncol 2016;5:379-387. 


\section{Cellular Physiology Cell Physiol Biochem 2018;46:471-481 and Biochemistry Published online: April 03, $2018 \quad \begin{aligned} & \text { DOI: 10.1159/000488615 } 2018 \text { The Author(s). Published by S. Karger AG, Basel } \\ & \text { www.karger.com/cpb }\end{aligned}$ \\ Yin et al.: miR-99a Enhances the Radiation Sensitivity}

5 Heist RS, Engelman JA: Snapshot: Non-small cell lung cancer. Cancer Cell 2012;21:448 e442.

6 Rosenzweig KE, Gomez JE: Concurrent chemotherapy and radiation therapy for inoperable locally advanced non-small-cell lung cancer. J Clin Oncol 2017;35:6-10.

7 Hoang T, Dahlberg SE, Schiller JH, Mehta MP, Fitzgerald TJ, Belinsky SA, Johnson DH: Randomized phase iii study of thoracic radiation in combination with paclitaxel and carboplatin with or without thalidomide in patients with stage iii non-small-cell lung cancer: The ecog 3598 study. J Clin Oncol 2012;30:616-622.

$>8$ Bartel DP: Micrornas: Genomics, biogenesis, mechanism, and function. Cell 2004;116:281-297.

-9 Benz F, Roy S, Trautwein C, Roderburg C, Luedde T: Circulating micrornas as biomarkers for sepsis. Int J Mol Sci 2016;17

10 Wang X, Chen X, Meng Q, Jing H, Lu H, Yang Y, Cai L, Zhao Y: Mir-181b regulates cisplatin chemosensitivity and metastasis by targeting tgfbetar1/smad signaling pathway in nsclc. Sci Rep 2015;5:17618.

11 Korpela E, Vesprini D, Liu SK: Microrna in radiotherapy: Mirage or mirador? Br J Cancer 2015;112:777-782.

12 Song Y, Zuo Y, Qian XL, Chen ZP, Wang SK, Song L, Peng LP: Inhibition of microrna-21-5p promotes the radiation sensitivity of non-small cell lung cancer through hmsh2. Cell Physiol Biochem 2017;43:12581272.

13 Mueller AC, Sun D, Dutta A: The mir-99 family regulates the DNA damage response through its target snf2h. Oncogene 2013;32:1164-1172.

14 Rane JK, Erb HH, Nappo G, Mann VM, Simms MS, Collins AT, Visakorpi T, Maitland NJ: Inhibition of the glucocorticoid receptor results in an enhanced mir-99a/100-mediated radiation response in stem-like cells from human prostate cancers. Oncotarget 2016;7:51965-51980.

15 Svoboda M, Sana J, Fabian P, Kocakova I, Gombosova J, Nekvindova J, Radova L, Vyzula R, Slaby O: Microrna expression profile associated with response to neoadjuvant chemoradiotherapy in locally advanced rectal cancer patients. Radiat Oncol 2012;7:195.

16 Gu W, Fang S, Gao L, Tan Y, Yang Z: Clinic significance of microrna-99a expression in human lung adenocarcinoma. J Surg Oncol 2013;108:248-255.

17 Sun M, Hong S, Li W, Wang P, You J, Zhang X, Tang F, Wang P, Zhang C: Mir-99a regulates ros-mediated invasion and migration of lung adenocarcinoma cells by targeting nox4. Oncol Rep 2016;35:2755-2766.

18 Clohessy JG, Reschke M, Pandolfi PP: Found in translation of mtor signaling. Cell Res 2012;22:1315-1318.

19 Guertin DA, Sabatini DM: Defining the role of mtor in cancer. Cancer Cell 2007;12:9-22.

20 Leiker AJ, DeGraff W, Choudhuri R, Sowers AL, Thetford A, Cook JA, Van Waes C, Mitchell JB: Radiation enhancement of head and neck squamous cell carcinoma by the dual pi3k/mtor inhibitor pf-05212384. Clin Cancer Res 2015;21:2792-2801.

21 Manegold PC, Paringer C, Kulka U, Krimmel K, Eichhorn ME, Wilkowski R, Jauch KW, Guba M, Bruns CJ: Antiangiogenic therapy with mammalian target of rapamycin inhibitor rad001 (everolimus) increases radiosensitivity in solid cancer. Clin Cancer Res 2008;14:892-900.

-22 Chen ZT, Zhao W, Qu S, Li L, Lu XD, Su F, Liang ZG, Guo SY, Zhu XD: Parp-1 promotes autophagy via the ampk/mtor pathway in cne-2 human nasopharyngeal carcinoma cells following ionizing radiation, while inhibition of autophagy contributes to the radiation sensitization of cne- 2 cells. Mol Med Rep 2015;12:1868-1876.

-23 Lee S, Kim D, Kang J, Kim E, Kim W, Youn H, Youn B: Surfactant protein b suppresses lung cancer progression by inhibiting secretory phospholipase a2 activity and arachidonic acid production. Cell Physiol Biochem 2017;42:1684-1700.

-24 Salim H, Akbar NS, Zong D, Vaculova AH, Lewensohn R, Moshfegh A, Viktorsson K, Zhivotovsky B: Mirna-214 modulates radiotherapy response of non-small cell lung cancer cells through regulation of p38mapk, apoptosis and senescence. Br J Cancer 2012;107:1361-1373.

25 Lee HC, Her NG, Kang D, Jung SH, Shin J, Lee M, Bae IH, Kim YN, Park HJ, Ko YG, Lee JS: Radiation-inducible mir-770-5p sensitizes tumors to radiation through direct targeting of pdz-binding kinase. Cell Death Dis 2017;8:e2693.

26 Wu D, Li Y, Zhang H, Hu X: Knockdown of Incrna pvt1 enhances radiosensitivity in non-small cell lung cancer by sponging mir-195. Cell Physiol Biochem 2017;42:2453-2466.

27 Yan B, Fu Q, Lai L, Tao X, Fei Y, Shen J, Chen Z, Wang Q: Downregulation of microrna 99a in oral squamous cell carcinomas contributes to the growth and survival of oral cancer cells. Mol Med Rep 2012;6:675-681.

-28 Li D, Liu X, Lin L, Hou J, Li N, Wang C, Wang P, Zhang Q, Zhang P, Zhou W, Wang Z, Ding G, Zhuang SM, Zheng L, Tao W, Cao X: Microrna-99a inhibits hepatocellular carcinoma growth and correlates with prognosis of 


\section{Cellular Physiology Cell Physiol Biochem 2018;46:471-481

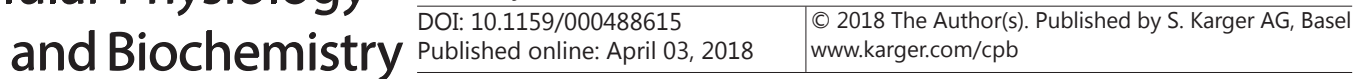 \\ Yin et al.: miR-99a Enhances the Radiation Sensitivity}

patients with hepatocellular carcinoma. J Biol Chem 2011;286:36677-36685.

29 Zhao J, Chen F, Zhou Q, Pan W, Wang X, Xu J, Ni L, Yang H: Aberrant expression of microrna-99a and its target gene mtor associated with malignant progression and poor prognosis in patients with osteosarcoma. Onco Targets Ther 2016;9:1589-1597.

-30 Choi EJ, Cho BJ, Lee DJ, Hwang YH, Chun SH, Kim HH, Kim IA: Enhanced cytotoxic effect of radiation and temozolomide in malignant glioma cells: Targeting pi3k-akt-mtor signaling, hsp90 and histone deacetylases. BMC Cancer 2014;14:17.

-31 Mehta M, Khan A, Danish S, Haffty BG, Sabaawy HE: Radiosensitization of primary human glioblastoma stem-like cells with low-dose akt inhibition. Mol Cancer Ther 2015;14:1171-1180.

32 Wang WJ, Long LM, Yang N, Zhang QQ Ji WJ, Zhao JH, Qin ZH, Wang Z, Chen G, Liang ZQ: Nvp-bez235, a novel dual pi3k/mtor inhibitor, enhances the radiosensitivity of human glioma stem cells in vitro. Acta Pharmacol Sin 2013;34:681-690.

33 Chang L, Graham PH, Hao J, Ni J, Bucci J, Cozzi PJ, Kearsley JH, Li Y: Acquisition of epithelial-mesenchymal transition and cancer stem cell phenotypes is associated with activation of the pi3k/akt/mtor pathway in prostate cancer radioresistance. Cell Death Dis 2013;4:e875.

-34 Ferlazzo ML, Bourguignon M, Foray N: Functional assays for individual radiosensitivity: A critical review. Semin Radiat Oncol 2017;27:310-315. 